\title{
OBSERVATIONAL EVIDENCE FOR NON-VELOCITY REDSHIFT IN STEPHAN'S QUINTET
}

\author{
C. BALKOWSKI, L. BOTTINELLI, P. CHA MARAUX, L. GOUGUENHEIM, \\ and J. HEIDMANN \\ Observatoire de Paris, Meudon, France
}

\begin{abstract}
Distance determinations of the high-redshift galaxy NGC 7319 in Stephan's Quintet, based on $21-\mathrm{cm}$ line observations, yield a value much smaller than the cosmological distance. At the $98.7 \%$ level, a part of the redshift of this galaxy which could amount to $5000 \mathrm{~km} \mathrm{~s}^{-1}$ is anomalous.
\end{abstract}

Stephan's Quintet is a tight group of five galaxies (Figure 1). Four of them have a redshift $c z \sim 6000 \mathrm{~km} \mathrm{~s}^{-1}$ while one, NGC 7320, has a much smaller $c z \sim 800 \mathrm{~km} \mathrm{~s}^{-1}$. To find out whether the galaxies in the Quintet are at the same distance from us, which would imply that they have anomalous redshifts, we observed two of them, the low redshift NGC 7320 and the high redshift NGC 7319, with the 21-cm line receiver at Nançay so as to be able to apply distance criteria entirely independent from redshifts (Balkowski et al., 1973).

Figure 2 shows the line profiles obtained. From them one derives the systemic redshifts (which agree with the optical determinations), the $21-\mathrm{cm}$ line flux densities $F_{H}$ and the total widths of the lines $W$.

Combining them with optical data for the apparent luminosities $l$ and apparent photometric diameters $a$, one may derive the values of four integral parameters:

- the intrinsic luminosities: $L \propto l D^{2}$, where $D$ is the distance;

- the linear diameters: $A \propto a D$;

- the neutral hydrogen masses: $M_{\mathrm{H}} \propto F_{\mathrm{H}} D^{2} ;$ and

- the indicative total masses: $M_{\mathrm{i}} \propto a D W^{2}$, to which one may add the morphological type $T$ as a fifth parameter.

We used $a, l$ and $F_{H}$ values corrected for inclination and absorption effects in galaxies following Heidmann et al. (1972). Our statistical studies (Balkowski, 1973) lead to various relations or correlations, of which those where $D$ enters are:

$$
\begin{aligned}
& M_{\mathrm{i}} / L\left(\propto D^{-1}\right) \text { vs } T, \\
& M_{\mathrm{H}} / M_{\mathrm{i}}(\propto D) \text { vs } \sigma_{\mathrm{H}},
\end{aligned}
$$

where $\sigma_{\mathrm{H}}$ is the mean neutral hydrogen surface density $\left(\propto F_{\mathrm{H}} / a^{2}\right)$,

$$
\begin{aligned}
& M_{\mathrm{H}} / M_{\mathrm{i}}(\propto D) \text { vs } T, \\
& \sigma_{\mathrm{H}} / A\left(\propto D^{-1}, \text { the quasi-volumic density }\right) \text { vs } T, \\
& L / A^{2.6}\left(\propto D^{-0.6}\right) \text { a constant. }
\end{aligned}
$$

These five relations are distance indicators. For example, observations of $a, W$ and $l$ lead to

$$
M_{\mathrm{i}} / L=\text { a numerical factor } \times a W^{2} l^{-1} D^{-1}
$$




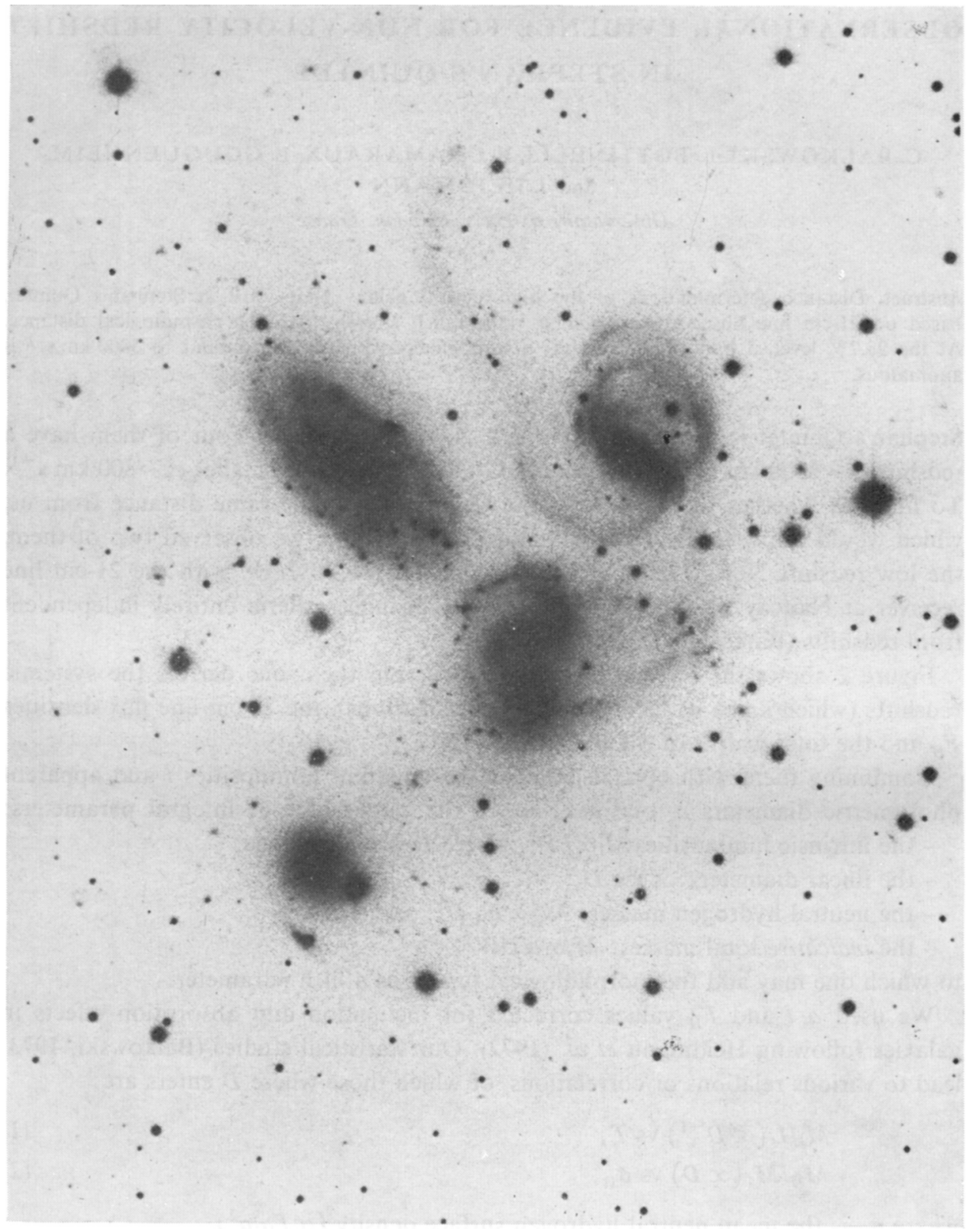

Fig. 1. Stephan's Quintet. NGC 7319 is at top right and NGC 7320 at top left. Mount Palomar 200 -in. photograph in red light by Arp (private communication). 

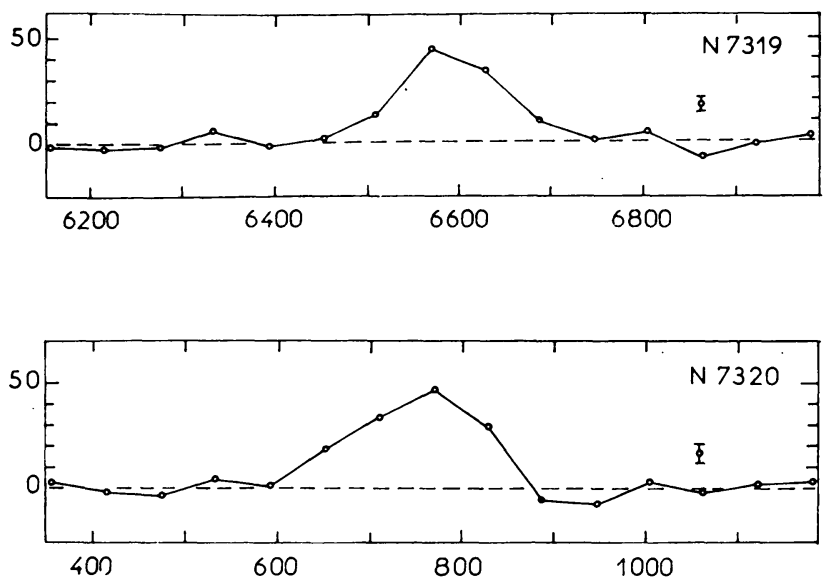

Fig. 2. 21-cm line profiles of NGC 7319 and 7320 . Ordinates are flux densities $\left(10^{-29} \mathrm{~W} \mathrm{~m}^{-2} \mathrm{~Hz}^{-1}\right)$; abscissae are redshifts $c z$ with respect to the Sun $\left(\mathrm{km} \mathrm{s}^{-1}\right)$. The error bars correspond to $\pm 1 \mathrm{rms}$ deviation.

and relation (1) gives the value of $M_{\mathrm{i}} / L$ corresponding to the type $T$ of the galaxy, hence $D$.

The $D$ values obtained by the five methods are shown in Figure 3, together with those obtained in the same way for the neighbouring large spiral NGC 7331. Our studies have shown that there are only three independent $D$ determinations among these five. For NGC 7319 the mean logarithmic value of the first three is

$$
\bar{D}=\left(22_{-9}^{+15} \text { m.e. }\right) \text { Mpc. }
$$

Our determinations are all in agreement with a common value $\sim 20 \mathrm{Mpc}$ for the three

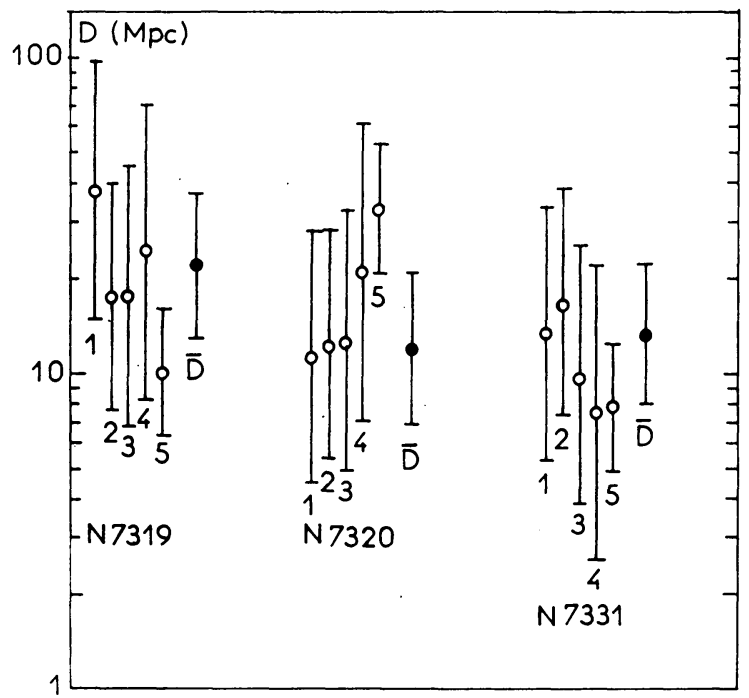

Fig. 3. Distance determinations in Mpc for NGC 7319, 7320 and 7331 with their mean errors from the five distance criteria. $D$ is the mean logarithmic value for the first three criteria. 
galaxies NGC 7319, 7320 and 7331, and thus support Arp's (1973) views about the Quintet.

From the redshift and a Hubble constant $H=(80 \pm 20) \mathrm{km} \mathrm{s}^{-1} \mathrm{Mpc}^{-1}$ (Heidmann, 1973), the cosmological distance of NGC 7319 would be $D_{\text {cosmo }}=\left(84_{-17}^{+28}\right) \mathrm{Mpc}$. Taking into account our error on $\bar{D}$ and the error on $H, D_{\text {cosmo }}$ differs from $\bar{D}$ by $2.2 \mathrm{rms}$ deviations, i.e. there is only a $1.3 \%$ chance that NGC 7319 is at $D_{\text {cosmo }}$. Then, at the $98.7 \%$ level, a part of the redshift of this galaxy which could amount to $5000 \mathrm{~km} \mathrm{~s}^{-1}$ is anomalous.

\section{References}

Arp, H. A.: 1973, Astrophys. J. 183, 411.

Balkowski, C.: 1973, Astron. Astrophys. 29, 43.

Balkowski, C., Bottinelli, L., Chamaraux, P., Gouguenheim. L., and Heidmann, J.: 1973, Astron. Astrophys. 25, 319.

Heidmann, J.: 1973, in G. Cayrel de Strobel and A. M. Delplace (eds.), 'L'âge des Etoiles', IAU Colloq. 17, XXXVII-1.

Heidmann, J., Heidmann, N., and de Vaucouleurs, G.: 1972, Mem. Roy. Astron. Soc. 75, 85, 105 and 121.

\section{DISCUSSION}

Allen: Could Dr Heidmann comment on the recent observations of $\mathrm{H}_{\mathrm{I}}$ in the Quintet made by Shostak (Astrophys. J. 187, 19, 1974; 189, L1, 1974) with the 300-ft NRAO telescope? Although Shostak observes line profiles for NGC 7319 and NGC 7320 similar to those of Dr Heidmann, he reaches the opposite conclusion, viz. that the two galaxies are most probably widely separated from each other.

Heidmann: I think, in fact, that Shostak's results are in good agreement with ours. When you plot his distance values for NGC 7319,7320 and 7331 on our diagram you see that they could all agree with a common distance $\sim 20 \mathrm{Mpc}$. Shostak's statement in his preprint according to which NGC 7319 is unlikely to be at the same distance as NGC 7320 is misleading, as the difference between his two distances is only one standard deviation.

Ekers: Could you remind us what the Hubble distances are for these galaxies and how your conclusions are affected by the value of the Hubble constant?

Heidmann: The statistics we used for evaluating the distances in Stephan's Quintet are completely independent of the Hubble constant; they do not use any distance deduced from redshift (see Balkowski, C. et al.: Astron. Astrophys. 25, 319, 1973).

Ekers: I find it puzzling that your distance determination can be independent of the Hubble constant, since that seems to imply that in other situations you could apply your procedure in reverse to determine the Hubble constant.

Heidmann: This is exactly one of our current programs.

Longair: In view of the complexity of the relations between the observed quantities, and their large dispersions, it would seem that the best way of estimating the errors in the correlations is by Monte Carlo techniques. Have you done this?

Heidmann: No, it was not necessary; we have statistics large enough to show that the distributions of the values are gaussian, so we used the standard procedure.

B. Peterson: The ratio you find for the radial velocities is about 8 . Is this right outside the range covered by the error bars?

Heidmann: Yes - the error bars correspond to a factor 1.7, much smaller than 8.

Lewis: Your use of average values of the various ratios implies that the objects are typical members of their classes. Have you any independent evidence to show that this is a valid assumption, e.g. that they have typical values of colour index, $B-V$ ?

Heidmann: Yes, we checked that point. We have three integral properties which are independent of distance: hydrogen mass-to-light ratio, mean surface hydrogen density and maximum rotational velocity; all of them have values falling inside the ranges of normal galaxies. For example, $M_{\mathrm{H}} / L_{0}=$ $=0.37$ (solar units) for NGC 7319 which, as you see from the figure (next page), is quite normal. 


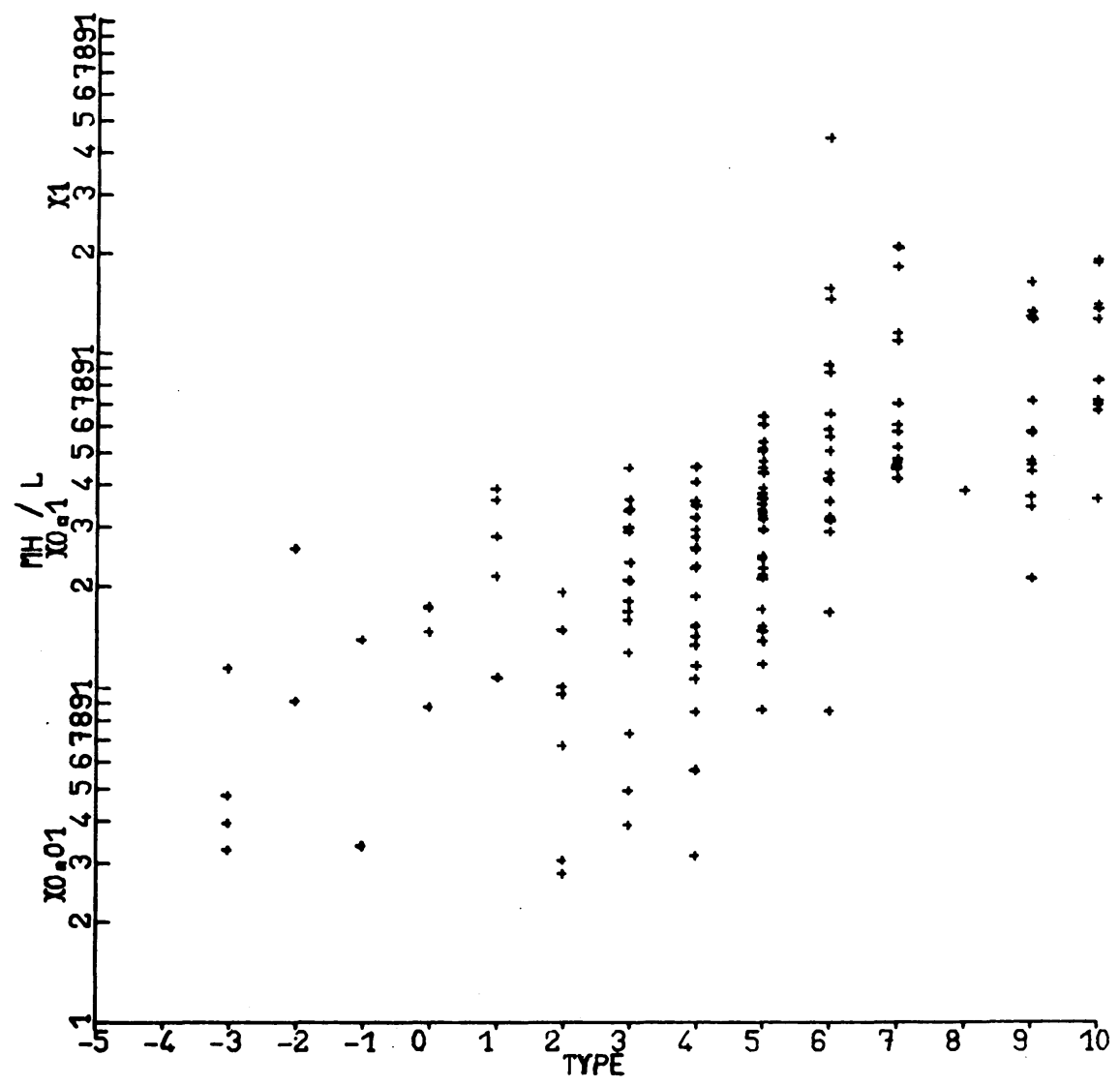

$M_{H} / L_{0}$ ratio in solar units versus morphological type $T$.

Sargent: I was planning to discuss Dr Heidmann's observations in my talk on the redshift question. My opinion is that the data as presented are consistent with either distance.

Arp: Two comments:

(i) The redshift distance of NGC 7319, using a Hubble constant of $50 \mathrm{~km} \mathrm{~s}^{-1} \mathrm{Mpc}^{-1}$, is $D=120 \mathrm{Mpc}$.

(ii) The Heidmann et al. distance for NGC 7319 is obtained from a comparison with data on field galaxies obtained by Balkowski and is not dependent on redshift distances. The Shostak distance is obtained by reference to Morton Roberts' data, some of which are based on a Hubble constant of $100 \mathrm{~km} \mathrm{~s}^{-1} \mathrm{Mpc}^{-1}$.

Oort: The fact that the galaxies in Stephan's Quintet lie in such a close group may well make them abnormal, so that the relations found for normal galaxies cannot safely be applied.

Heidmann: Yes, this may be so but, as I said, the three integral properties $M_{\mathrm{H}} / L_{0}, \sigma_{\mathrm{H}}$ and $V_{m}$ are normal, so we have nothing to compel us to assume they are abnormal. Even the photometric diameter of NGC 7319 appears normal, as is discussed in our paper.

Oort: On the other hand, the result you get is so improbable that I would try any way of escape first.

G. de Vaucouleurs: Has Dr van den Bergh derived luminosity classes for any member of the Quintet?

van den Bergh: The fact that the galaxies in Stephan's Quintet (?) are interacting makes it very difficult to assign unambiguous luminosity classifications to them.

Allen: Of course, the interesting thing to know is: just where is the gas located at the two different 
redshifts, and what is its dynamics? Dr W. Sullivan and I (in preparation) have mapped the distribution and velocity field of $\mathrm{H}_{\mathrm{I}}$ in NGC 7320 with the Westerbork telescope. Our conclusion is that the distribution and motions of the $\mathrm{H}_{\mathrm{I}}$ are quite reasonable for a dwarf galaxy in circular differential rotation. We put an upper limit of $20 \mathrm{~km} \mathrm{~s}^{-1}$ on any peculiar gas velocities caused by tidal effects of the other four Quintet members on the outer parts of NGC 7320. The minimum separation which one can infer from this observation is quite model dependent, but we can say that if we assume the five galaxies have been close to each other for at least one rotation period of NGC 7320 then the minimum separation between the latter galaxy and the other four members would be of order ten times the radius of NGC 7320.

Peebles: I have a philosophical question. If you assume that some galaxies have a non-Doppler redshift, does it make sense to assume that other physical parameters, such as transition rates, are the same as in normal galaxies, so that there is just this one aberration?

Toomre: Philosophical questions cannot be answered! 